\title{
Ingreso, permanencia y egreso de personas con diversidad funcional en la educación superior
}

\section{Entry, permanence and exit of people with functional diversity in higher education}

http://dx.doi.org/10.17981/cultedusoc.13.1.2022.08

Recibido: 2 de junio de 2020. Aceptado: 3 de diciembre de 2020. Publicado: 24 de enero de 2022.

\author{
Sandra Marcela Zúñiga-Bolívar (i) \\ Institución Universitaria Escuela Nacional del Deporte. Cali (Colombia) \\ sandra.zuniga@endeporte.edu.co \\ Faisury Mejía-Botina \\ Institución Universitaria Escuela Nacional del Deporte. Cali (Colombia) \\ faisury.mejiab@endeporte.edu.co \\ Daniela Andrea Grijalba-Pantoja \\ Institución Universitaria Escuela Nacional del Deporte. Cali (Colombia) \\ danyandrea1999@gmail.com \\ María Camila Gamboa-Gómez \\ Institución Universitaria Escuela Nacional del Deporte. Cali (Colombia) \\ gamboagomezmariacamila@gmail.com
}

Para citar este artículo:

Zúñiga-Bolívar, S., Mejía-Botina, F., Grijalba-Pantoja, D. y Gamboa-Gómez, M. (2022). Ingreso, permanencia y egreso de personas con diversidad funcional en la educación superior. Cultura, Educación y Sociedad, 13(1), 125-142. DOI: http://dx.doi.org/10.17981/ cultedusoc.13.1.2022.08

\section{Resumen}

Introducción: La educación superior con un enfoque educativo basado en la valoración de la diversidad funcional y el reconocimiento de la existencia de barreras, permite a las personas ser incluidas efectivamente en la sociedad. Se analizaron factores como falencias y potencialidades que pueden influir en la vida universitaria de personas con o sin discapacidad física, mental o sensorial, para el acceso, la permanencia, participación y el egreso oportuno de la misma. Metodología: Se efectuó un estudio descriptivo de corte transversal, mediante análisis univariado y bivariado, que relaciona variables sociodemográficas con el nivel de funcionalidad en cuanto a la capacidad de desempeño y participación, considerando los 6 dominios del cuestionario para la Evaluación de la Discapacidad de la Organización Mundial de la Salud (Whodas 2.0). Resultados y Discusión: Se evidencia la importancia de desarrollar un diagnóstico inicial de los estudiantes que ingresen a las universidades con y sin discapacidad que limitan su participación y desempeño educativo, con el fin de potenciar sus habilidades, capacidades e intereses, para alcanzar un desempeño óptimo. Conclusiones: Se evidencian dificultades en el grado de funcionalidad de los dominios evaluados por el Whodas 2.0, lo que puede incrementar los porcentajes de deserción de los estudiantes principalmente en los primeros semestres.

Palabras clave: Educación superior; accesos a la educación; proceso de aprendizaje; política educacional; deserción escolar; educación inclusiva

\section{Abstract}

Introduction: Higher education with an educational approach based on the appreciation of functional diversity and the recognition of barriers allows people to be effectively included in society. To this end, we analyzed factors such as shortcomings and potentialities, which can influence the university life of people with or without physical, mental, or sensory disabilities, for access, permanence, participation and timely discharge of the same. Methodology: A descriptive cross-sectional study was carried out using univariate and bivariate analysis, which relates sociodemographic variables to the level of functionality in terms of performance capacity and participation, considering the 6 domains of the World Health Organization Disability Assessment Questionnaire (Whodas 2.0). Results and discussion: Noting the importance of developing an initial diagnosis of students entering universities with and without disabilities that limit their participation and educational performance, in order to enhance their skills, capacities and interests, to achieve optimal performance. Conclusions: Difficulties are evident in the grade of functionality of the domains evaluated by Whodas 2.0, which can increase the dropout rates of students, mainly in the first semesters.

Keywords: Higher education; access to education; learning process; educational policy; school dropout; inclusive education 


\section{INTRODUCCIÓN}

La Educación Superior en Colombia es un servicio público cultural inherente a la finalidad social del Estado, que posibilita el desarrollo de potencialidades del ser humano de una forma integral (Ley 30, 1992, art. 1; art. 2). Ahora bien, respecto a la educación inclusiva, Acedo y Opertti (2012) refieren la importancia de favorecer una cultura educativa inclusiva, donde se les brinden a las personas calidad de oportunidades efectivas y equitativas, garantizando la participación y el aprendizaje de manera conjunta con los demás.

En virtud de ello, la inclusión educativa constituye un enfoque educativo basado en la valoración de la diversidad funcional, como elemento enriquecedor del proceso enseñanza aprendizaje y, en consecuencia, favorecedor del desarrollo humano. Por otra parte, hace referencia a las transformaciones de la educación general y de las instituciones educativas para dar respuesta equitativa y de calidad a la diversidad (Parra, 2011). De este modo, desarrollar un modelo universitario basado en los principios de la educación inclusiva, implica reconocer la existencia de barreras familiares, sociales, culturales y educativas, que limita a las personas con discapacidad ser incluidas efectivamente en la sociedad (Moriña, Cortés y Molina, 2017).

El concepto de educación inclusiva puntualiza una academia igual para todos, independientemente de las capacidades, habilidades y competencias del estudiantado; dando respuesta al sistema educativo como una estrategia para abordar situaciones de desventaja y vulnerabilidad que, por diferentes motivos, pueden afectar la vida de las personas y brindando las mejores oportunidades educativas posibles a todo el alumnado. En este sentido, la educación inclusiva hace énfasis en las barreras del sistema, tanto culturales, administrativas, políticas y prácticas, resaltando la necesidad de asegurar la presencia, participación y el aprendizaje de toda la comunidad estudiantil (Menéndez, Pevida y Dopico, 2020). Por consiguiente, podemos afirmar que la diversidad es aceptar el derecho de cada individuo a ser distinto y a ejercer ese derecho sin distinciones de ninguna clase. En un sentido, no de exclusión, negación, rechazo, marginación o discriminación; sino de reconocimiento, inclusión y aceptación de lo diverso como parte de un mismo organismo comunitario.

Por las consideraciones anteriores, se realizó una investigación titulada "Caracterización desde la funcionalidad de los estudiantes de primer semestre de 2018-1 de la facultad de salud y rehabilitación de una institución universitaria de la ciudad de Cali, Colombia"; obteniendo a partir de una encuesta estructurada y validada por la Organización Mundial de la Salud (OMS, 2010), el nivel de funcionamiento en los 6 dominios de la vida, los cuales se describen a continuación:

La cognición es la capacidad que permite al ser humano desarrollar una vida sin dificultad, resolviendo problemas y situaciones, manteniendo un óptimo aprendizaje y procesando oportunamente la información del ambiente para recordarla y usarla posteriormente (Gutiérrez y Guzmán, 2017); donde intervienen las funciones ejecutivas que posibilitan llevar procesos mentales necesarios para la planificación, organización, regulación y evaluación de un comportamiento. Así mismo, los procesos cognitivos hacen referencia a la comprensión lectora, al análisis, a la síntesis y a la metacognición que permite o facilita la incorporación de nuevos conocimientos (Velásquez, 2019). 
Por otra parte, el dominio de la movilidad es definido como toda habilidad que tiene la persona para efectuar todas las actividades que se encuentran inmersas dentro del traslado de un lugar a otro en un espacio determinado como, el desplazamiento a diferentes lugares en diversos terrenos de manera independiente con o sin algún tipo de ayuda técnica, permitiendo así su participación en actividades y acceso a diferentes espacios públicos, tomando como base la infraestructura universitaria. No obstante, según Jirón (2015):

La movilidad hace referencia al desplazamiento físico de las personas desde un espacio a otro en el territorio, y relaciona con los significados que estas movilidades generan en términos de sus consecuencias sociales, culturales, económicas, físicas, entre otras y, por otro lado, las experiencias de las personas sobre estos desplazamientos (p. 50).

En cambio, González y Giraldo (2019) definen la movilidad como un derecho el cual permite la conexión de relaciones sociales que se dan a raíz del intercambio de flujos de personas, bienes o servicios, a partir de un espacio específico, al cual se le aporta un sentido, según lo particular de este y dependiendo del contexto.

El tercer dominio, cuidado personal, hace alusión a la competencia que tiene el individuo para realizar de manera autónoma, tareas de autocuidado que le brinden bienestar y calidad de vida. En este sentido, se define el autocuidado como cuidar de la existencia personal, la salud y la apariencia personal; por lo que compone un conjunto de conductas accesibles para cualquier persona, como ejercitarse, tener una buena nutrición, cuidar los hábitos de salud o relajarse (Campos-Garcái, Oliver, Tomás, Galiana y Gutiérrez, 2018). Por consiguiente, el autocuidado tiene un matiz diferente en función de la cultura y son trasmitidas de una generación a otra, varían en dependencia del contexto histórico-social, nivel económico, status social, color de la piel, edad, sexo, entre otros; y es así como, se erige una estrategia para la prevención de enfermedades y promoción de conductas saludables, por medio del cual se puede contribuir al cuidado integral del ser humano, convirtiéndolo en un gestor de su propio cuidado, ganando autonomía y mayor control sobre si, para el fomento y conservación de su propia salud (Cancio-Bello, Lorenzo y Alarcó, 2020).

En consecuencia, estos dominios mencionados previamente se encuentran relacionados con el dominio de actividades cotidianas, el cual comprende todas las tareas que la persona realiza de manera habitual y durante el día (auto mantenimiento, actividades domésticas, desplazamientos, compras, etc.) (Olivera, Salvador y Elorduy, 2015). Es decir, todas aquellas que realiza la persona dentro de su diario vivir, desde las diferentes áreas de ocupación como básicas, instrumentales, trabajo, estudio, descanso y sueño, ocio y tiempo libre.

Estos dominios permiten a la persona desarrollar una imagen de su esquema corporal relacionado directamente con algunos factores emocionales como el autoconcepto y autoestima, los cuales brindan seguridad al individuo para una adecuada participación e interacción social con pares u otras personas durante el ciclo de la vida. El relacionarse con otras personas, según Garza (2015), es la capacidad que tiene el ser humano de establecer y generar vínculos con otro sujeto o grupo social los cuales actúan bajo unos valores y normativas, con el fin de dar respuesta a diversas necesidades en común. Esto se debe, 
a que constituir lazos emocionales cercanos con otros seres humanos es una necesidad, es por esto por lo que la participación en sociedad implica el involucramiento del individuo en actividades de convivencia social, ya sea de tipo: deportivo, artístico o cultural, acción en grupos organizados, entre otros. La convivencia social puede llevarse a cabo de manera presencial o virtual, y puede aplicarse a diferentes contextos y/o escenarios (Rivera, Martínez, González y Salazar, 2016). En consecuencia, el relacionarse con otras personas le provee el atributo al individuo para tener la oportunidad de participar en la sociedad, gozando de diferentes tipos de actividades, que se desarrollan dentro de una población o comunidad, en un estado político como por ejemplo, la participación política, cultural, religiosa y ciudadana, sin ningún tipo de barreras políticas, actitudinales, sociales y físicas.

Por otra parte, Borzone (2017) plantea que:

La vida universitaria involucra niveles adecuados de disciplina, planificación, manejo del tiempo, tolerancia a la frustración y persistencia; por este motivo, la población estudiantil debe enfrentarse a múltiples y complejos desafíos académicos o institucionales, a sistemas de enseñanza y evaluación, a la toma de decisiones vocacionales, al aprovechamiento de recursos institucionales en general, y a patrones de relaciones interpersonales con familiares, profesores y pares (p. 267).

Al respecto, Alharthi (2019) refiere que cada año, varios millones de estudiantes de todo el mundo realizan el cambio de la educación secundaria a la superior. Sin embargo, existe una diferencia considerable entre ser estudiante en la escuela y en la universidad, y las investigaciones anteriores han puesto de relieve las dificultades que enfrentan los estudiantes universitarios de primer año durante su fase de transición, indicando una alta tasa de deserción entre los estudiantes de primer año.

De ahí que, el estudiante que inicia la vida universitaria afronta el impacto que le genera los problemas en el desempeño que se suman al efecto procedente de la transición y que en varias ocasiones se traducen en la pérdida de la matrícula. Entre las numerosas dificultades entre las cuales puede atravesar el alumnado se puede considerar las falencias que puede arrastrar del nivel medio, la falta de preparación del joven en relación con lo que proyecta para su vida futura, la falta de herramientas para enfrentar el estudio universitario al igual que hábitos de estudio e incluso, en muchos casos, la necesidad de incorporarse simultáneamente al mundo laboral (Moreno, Chiecher y Paoloni, 2020).

En este sentido, la vida universitaria demanda al estudiante administrar autónomamente su tiempo, pensamiento crítico y capacidades que hacen parte de las exigencias que la sociedad requiere de los profesionales. Lo que implica un proceso de transición e incorporación a un nuevo mundo social. Además, "deben afrontar una serie de tareas y exigencias complejas, cuya ejecución implica alcanzar el desarrollo de su identidad y, a su vez, una adaptación adecuada al contexto" (Almeida, Ferreira \& Soares, citados por Borzone, 2017). Por consiguiente, incluye las relaciones interpersonales, aspectos de comunicación, además de la relación del estudiante con la universidad, no como establecimiento meramente docente, sino como experiencia completa, que facilita espacios tanto educativos como de esparcimiento y cuya oferta incluye, en algunos casos, servicios 
recreativos, espirituales, de salud física y mental (Borzone, 2017). En la que también es fundamental la historia de vida de los estudiantes, pues esta incide en el desarrollo de su talento cognitivo, aptitudes y habilidades para participar no solo en la vida universitaria, sino en las actividades cotidianas en un entorno físico y social.

El estudio realizado se fundamentó bajo la Clasificación Internacional del Funcionamiento de la Discapacidad y de la Salud-CIF, que fue adoptada en el año 2001 por la Asamblea Mundial de la Salud en virtud de la resolución WHA-54.21. "Esta clasificación se apoya en un enfoque sistémico de la discapacidad, a través del concepto de funcionamiento humano y proporciona un marco de referencia internacional y estandarizado para la recopilación de datos sobre la discapacidad" (Cuenot, 2018).

De manera que, la CIF se basa en el concepto de funcionamiento como:

Un término genérico para designar los aspectos positivos de la interacción, entre un individuo que tiene un problema de salud y los factores contextuales frente a los que evoluciona. Asimismo, en este marco de referencia, la discapacidad no es equivalente a la deficiencia, ni a una limitación de tipo funcional. La discapacidad es una restricción de la participación social, que corresponde a los resultados negativos de la interacción entre las características individuales y las características del entorno (Cuenot, 2018, p. 2).

Por esta razón; la investigación se efectuó con el fin de caracterizar detalladamente a la población en estudio, logrando identificar falencias y potencialidades que pueden influir en la vida universitaria. A partir de esto, institucionalmente se puede podría promover un desarrollo académico y social de los estudiantes con y sin discapacidad, a través de medios que faciliten la participación y desarrollo de programas de educación inclusiva, considerando las diferentes políticas institucionales que respondan a las características y necesidades individuales de las personas para favorecer el ingreso, la continuidad y el egreso oportuno de la población estudiantil.

\section{MEtodología}

El presente estudio es descriptivo de corte transversal, llevado a cabo en una institución universitaria de la ciudad de Cali (Colombia), con estudiantes de primer semestre del periodo 2018-1, de los programas ofertados por la facultad de Salud y Rehabilitación, el cual permitió describir las características, el nivel de funcionalidad y discapacidad, además de evidenciar los factores que influyen en el ingreso, la permanencia y el egreso de dichos estudiantes.

Nivel de funcionalidad y discapacidad: Entendida como, cada función de un sujeto, a nivel corporal, de la persona o de la sociedad, en la cual se establece una evaluación a nivel operacional, y de discapacidad como "una disminución en cada uno de los dominios de funcionamiento". En consecuencia, se utilizó el Cuestionario de la Organización Mundial de la Salud, WHODAS 2.0 (Üstün, Kostanjsek, Chatterji, Rehm \& World Health Organization, 2010).

La población ya mencionada se tomó a través de un muestreo no probabilístico por conveniencia con aquellos estudiantes que se encontraran cursando activamente el primer semestre del periodo 2018-1, además que aceptaran voluntariamente participar, los anteriores se distribuyeron de acuerdo a la Tabla 1. 
TABLA 1.

Población de Muestra.

\begin{tabular}{ll}
\hline \multicolumn{1}{c}{ Programa Académico - Semestre 1} & Número de Estudiantes matriculados en el periodo 2018-1 \\
\hline Terapia ocupacional & 30 \\
Fisioterapia & 100 \\
Nutrición y Dietética & 88 \\
Total de la población & 218 \\
\hline
\end{tabular}

Fuente: Elaboración propia.

\section{Criterios para la participación de los encuestados}

La población estuvo constituida por estudiantes pertenecientes a una institución universitaria de la ciudad de Cali, teniendo en cuenta lo siguiente:

\section{- Criterios de Inclusión}

- Estudiantes con discapacidad física, mental, sensorial o sospecha de esta que se encuentren caracterizados por la institución.

- Estudiantes sin discapacidad que cumplan con criterios de comparabilidad relacionados con sexo, edad, estado civil, programa académico y semestre al cual pertenecen.

- Estudiantes matriculados en el periodo 2018 de primer semestre de la facultad de salud y rehabilitación.

- Estudiantes que acepten participar voluntariamente en el estudio.

- Criterios de exclusión

- No se tendrán en cuenta los estudiantes de postgrado de la facultad de salud y rehabilitación que realizan la Especialización de Actividad Física.

- Escuela Nacional del Deporte (END, 2018).

\section{Recolección de la información}

Esta investigación contó con la participación de tres (3) encuestadoras quienes fueron capacitadas por personal experto para el conocimiento de los aspectos generales del estudio e implementación del instrumento, por medio de ejercicios de aplicación. Se utilizó el instrumento WHODAS 2.0 (Üstün et al., 2010), este instrumento, permite medir la salud y la discapacidad en la población y en la práctica clínica, proporcionando un perfil y una medición total del funcionamiento y la discapacidad, es una herramienta fiable y aplicable a todas las poblaciones adultas. Consta de 6 dominios: Cognición-Movilidad-Cuidado personal-Relaciones con otras personas-Actividades cotidianas-Participación. 


\section{Procesamiento de la información}

Digitación de los datos: Los datos recolectados fueron almacenados en bases de datos construidas en el programa Microsoft Excel, con un seguimiento semanal, con el fin de verificar que la información de los cuestionarios no presentara ninguna inconsistencia. Estos datos fueron organizados por cada uno de los 6 dominios que establece el WHODAS 2.0 (cognición, movilidad, cuidado personal, relaciones, Actividades de la Vida Diaria-AVD y participación). Partiendo de lo anterior, se establecieron promedios y porcentajes que permitió identificar el nivel de dificultad de la población evaluada, dando cuenta de la situación actual de los estudiantes de primer semestre 2018-1 de la facultad de salud y rehabilitación en diferentes áreas de desempeño ocupacional.

\section{Plan de análisis}

Para realizar la caracterización de la población se utilizó una estadística descriptiva, a través de un análisis univariado en donde se elaboraron tablas, clasificando las variables cuantitativas a partir de la estimación de promedios, medianas y desviación estándar. En cuanto a las variables cualitativas se estimaron proporciones en cada dominio contemplado en la investigación.

Los seis (6) dominios presentados en la evaluación WHODAS 2.0 son: Cognición, movilidad, cuidado personal, relaciones, actividades de la vida diaria, participación; los cuales adoptaron los siguientes valores de respuesta establecidos por la Organización Mundial de la Salud (Üstün et al., 2010), como escala politómica: ninguna = 1 , leve $=2$, moderada $=3$, severa $=4$ y extrema $=5$. Para la construcción de la variable respuesta o dependiente denominada nivel de funcionalidad global, se sumaron todos los dominios contemplados en el instrumento de recolección de datos (WHODAS 2.0). Posteriormente, se multiplicó por 100 y se dividió en 106, donde se tomó en cuenta los ítems de mayor puntuación en los dominios, incluyendo aquellos relacionados con el trabajo remunerado. Por otra parte, las variables independientes propuestas en este estudio se relacionaron con la edad, sexo, nivel de escolaridad, ocupación, estado civil, situación laboral y años estudiados.

Para encontrar la relación entre las variables personales y el nivel de funcionalidad global, se establecieron unas categorías e intervalos denominados: 1) sin dificultad, el cual corresponde a toda la población ubicada con $0 \%$; dificultad mínima entre $0.9 \%$ $20.8 \%$ y dificultad moderada entre $21 \%-100 \%$ (Tabla 3). Lo anterior, se desarrolló a través de la ejecución de un análisis bivariado, en donde se establecieron relaciones utilizando la prueba exacta de Fisher y la prueba de chi- cuadrado dependiendo la naturaleza de las variables. A partir de lo anterior, se seleccionaron las variables cuya significancia estadística fuera $\mathrm{p}<0,05$, las cuales fueron consideradas para la explicación de los resultados finales que den cuenta de esta investigación. Cabe resaltar que estos procedimientos se realizaron con el programa estadístico STATA 12 (StataCorp, 2011). 


\section{RESUltados}

Es relevante que las Instituciones educativas y universidades caractericen desde primer semestre los estudiantes que llegan al plantel con y sin discapacidad, para que, a partir de un diagnóstico inicial, se puedan potenciar las habilidades, capacidades e intereses con las que cuentan los estudiantes, brindándoles los apoyos necesarios con el fin de favorecer el ingreso, permanencia y egreso satisfactorio en la educación superior. Por consiguiente, en este estudio se logró evidenciar que $85.2 \%$ de los estudiantes participaron activamente, siendo la mayoría de género femenino con un $71.2 \%$. Por otra parte, menos de la mitad de la población fueron hombres con $28.7 \%$. Además, Asimismo, quedó registrado que una mínima parte de la población que participó del estudio optó por no dar respuesta a este ítem (Tabla 2).

Respecto a la edad se encontró que el promedio etario de los estudiantes partícipes fue 19 años y la mediana de 26 años; ubicándose de esta manera en un $76.4 \%$ en rangos de edad clasificados entre 15-19 años; menos de la mitad entre $20-29$ años con $21.2 \%$ y, solo $1.4 \%$ en el rango de 30-36 años. Por otra parte, 1\% de los participantes no respondieron a la pregunta relacionada con estas variables. Con relación al nivel de escolaridad se evidenció que la moda en el ítem años de estudio se encontraba entre los 11 y 13 años, siendo esta una serie multimodal; por tanto $89.4 \%$ de la población encuestada señaló tener años de estudio que oscilan entre los 11 y 15 años, mientras $6.1 \%$ de la población estudió entre 16 y 23 años. No obstante el $4.2 \%$ prefirió no brindar información y $0.2 \%$ de la población registró información inadecuada (Tabla 2).

TABLA 2.

Caracterización sociodemográfica de estudiantes de 1er semestre.

\begin{tabular}{|c|c|c|c|}
\hline \multicolumn{2}{|c|}{ Variables Sociodemográficas } & Frecuencia Absoluta & \multirow{2}{*}{$\begin{array}{l}\text { Frecuencia Relativa } \\
71.2 \%\end{array}$} \\
\hline \multirow{2}{*}{ Sexo } & Mujer & 151 & \\
\hline & Hombre & 61 & $28.7 \%$ \\
\hline \multirow{4}{*}{ Edad } & 15 a 19 & 162 & $76.4 \%$ \\
\hline & 20 a 29 & 45 & $21.2 \%$ \\
\hline & 30 a 36 & 3 & $1.4 \%$ \\
\hline & No responde & 2 & $0.9 \%$ \\
\hline \multirow{3}{*}{$\begin{array}{l}\text { Años De } \\
\text { Estudio }\end{array}$} & 11 a 15 & 190 & $89.4 \%$ \\
\hline & 16 a 23 & 13 & $6 \%$ \\
\hline & No responde & 9 & $4 \%$ \\
\hline \multirow{4}{*}{ Estado Civil } & Soltero (a) & 192 & $90.5 \%$ \\
\hline & Separado & 10 & $4.7 \%$ \\
\hline & Otro estado civil & 5 & $2.3 \%$ \\
\hline & No responde & 5 & $2.3 \%$ \\
\hline \multirow{5}{*}{$\begin{array}{l}\text { Principal } \\
\text { Situación } \\
\text { Laboral }\end{array}$} & Estudiante & 189 & $89.1 \%$ \\
\hline & Trabajo remunerado & 10 & $4.7 \%$ \\
\hline & Desempleado & 2 & $0.9 \%$ \\
\hline & Otra situación laboral & 5 & $3 \%$ \\
\hline & No responde & 4 & $1.9 \%$ \\
\hline
\end{tabular}

Fuente: Elaboración propia. 
En cuanto al estado civil, se halló que el 90.5\% de la muestra nunca había estado casado(a) y un porcentaje del $4.7 \%$ de los participantes respondieron estar separado(a). Por último, lo que corresponde a la situación laboral, el 89.1\% de la población eran estudiantes; ya que uno de los principales requisitos para participar en la prueba, era que estuviesen cursando activamente primer semestre en el periodo 2018-1. Por otra parte, el 7\% de esta población además de estudiar, trabaja y muchos de ellos realizaban trabajos independientes; por tanto, recibían una remuneración para solventar sus necesidades básicas (Tabla 2).

En un segundo momento, se realizó un análisis univariado que permitió evidenciar el estado actual de los estudiantes en cada uno de los seis dominios evaluados por el Whodas 2.0. El primero se encuentra relacionado con los procesos cognitivos, en el cual se encontró que más de la mitad de los estudiantes que participaron en esta investigación presentan una dificultad leve con un 63.1\%, y aproximadamente 25\% mostró una dificultad moderada. Por otra parte, $5 \%$ refirió tener una dificultad severa. Por consiguiente, estos altos porcentajes en los diferentes niveles de dificultad son un factor alarmante; puesto que pueden estar teniendo un desempeño académico no adecuado, lo cual puede relacionarse con la deserción y el egreso no oportuno de los mismos (Figura 1).

El segundo dominio evaluado fue la movilidad, donde se evidenció que aproximadamente la mitad de la población no presenta dificultades para el acceso a los diferentes espacios en las instalaciones de la institución. Sin embargo, 37.9\% de los encuestados refirieron tener una dificultad leve en dicho dominio, y $12.16 \%$ manifestaron tener una dificultad moderada, severa o extrema; lo cual significa otro factor alarmante debido a que puede ser una de las causas del acceso, la permanencia y el egreso oportuno del estudiantado (Figura 1).

Luego, se evaluó el dominio de cuidado personal, logrando dar cuenta que más del 70\% de la población en estudio no presentó ningún tipo de dificultad en el desempeño de estas actividades. No obstante, $17.7 \%$ presentan una dificultad leve en actividades como baño, vestido y alimentación que evalúa el Whodas; y por último, 5.3\% indicaron presentar una dificultad moderada o extrema, repercutiendo de tal manera su desempeño ocupacional (Figura 1).

Posteriormente se evaluó el cuarto dominio de relaciones con otras personas, donde alrededor del 40\% de los estudiantes no tienen ninguna dificultad para relacionarse. Sin embargo, más del 60\% si manifestó tener algún tipo de dificultad leve, moderada o severa, donde se ve afectada de manera directa o indirecta el establecimiento de relaciones o nuevos vínculos con otros; el cual repercute en gran instancia en el nivel de funcionalidad y rendimiento académico del estudiantado (Figura 1).

En lo correspondiente al quinto dominio de las actividades de la vida diaria, 73\% de los estudiantes manifestaron tener algún tipo de dificultad para la ejecución de estas actividades, siendo una de las principales causas, las extensas jornadas de estudio y alta cantidad horaria del proceso de formación académico universitario (Figura 1).

En cuanto al último dominio que hace referencia a la participación en la sociedad, se obtuvo que más de la mitad de la población, presenta dificultad leve en los procesos de participación evaluados, como actividades en comunidad por barreras y obstáculos en el medio físico y social, características económicas o psicosociales. Por otra parte, aproximadamente $20 \%$ presentan dificultad moderada o severa, lo cual implica una falta de participación en las diferentes actividades de la vida universitaria donde se debe asumir un rol proactivo (Figura 1). 


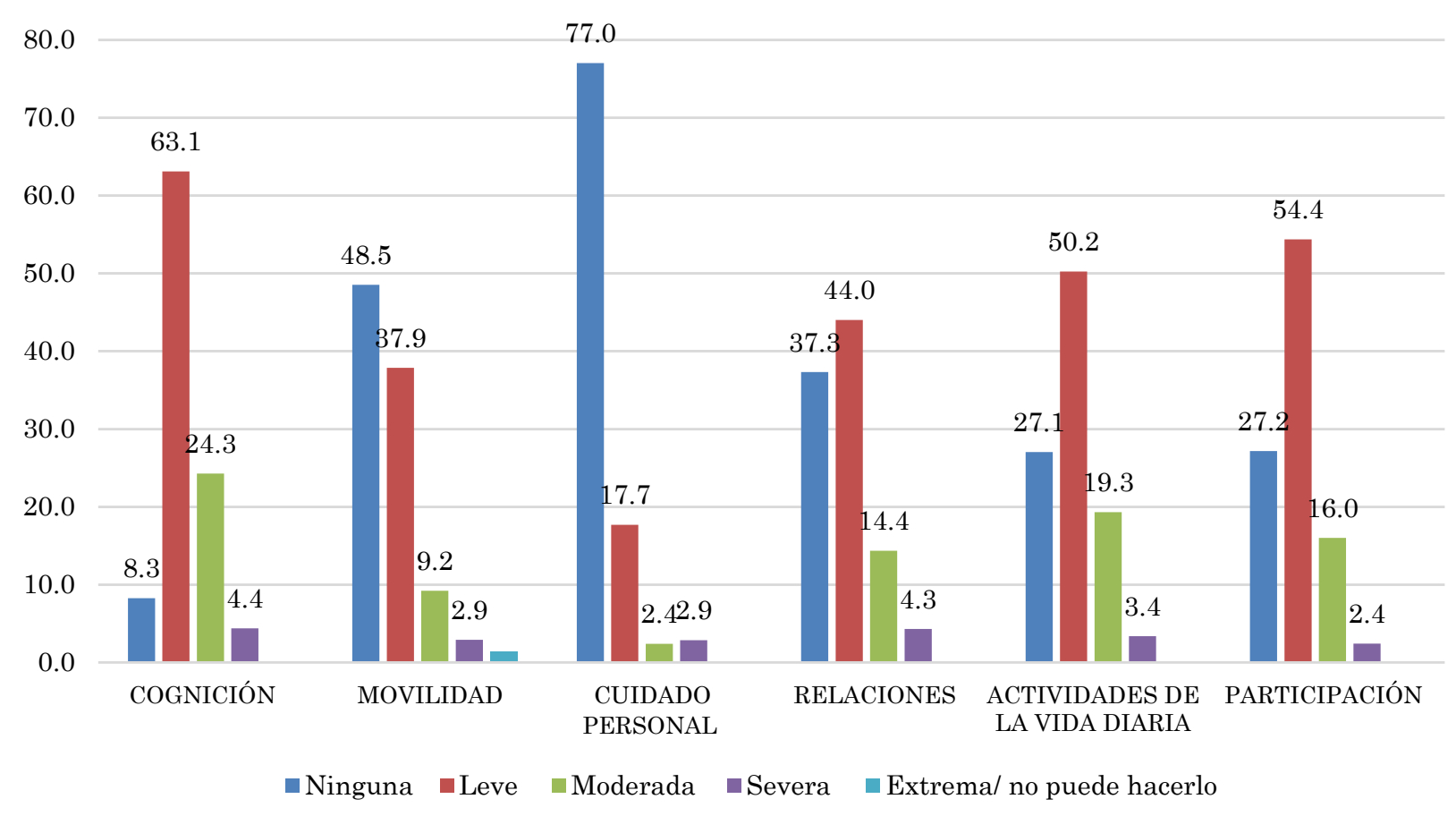

Figura 1. Estado actual de los estudiantes de primer semestre de la facultad de Salud y Rehabilitación en los dominios evaluados por el Whodas 2.0.

Fuente: Elaboración propia.

Por otra parte, en un tercer momento se desarrolló un análisis bivariado que permitió conocer si los factores sociodemográficos influyen en el nivel global de funcionalidad de los estudiantes universitarios, a través de la aplicación de la prueba de asociación Fisher, evidenciándose que ninguna de las variables anteriormente mencionadas, se relacionan estadísticamente con el nivel de funcionalidad de los estudiantes, obteniendo un valor $p$ mayor a 0.05 (Tabla 3).

TABLA 3.

Análisis bivariado de variables sociodemográficas y nivel de funcionalidad de los estudiantes.

\begin{tabular}{|c|c|c|c|c|c|c|c|c|}
\hline \multirow{2}{*}{\multicolumn{2}{|c|}{ Variables Sociodemográficas }} & \multicolumn{6}{|c|}{ Nivel de Funcionalidad } & \multirow{3}{*}{$\begin{array}{l}\text { Valor P } \\
0.178\end{array}$} \\
\hline & & \multicolumn{2}{|c|}{ Sin Dificultad } & \multicolumn{2}{|c|}{ Dificultad Mínima } & \multicolumn{2}{|c|}{ Dificultad Moderada } & \\
\hline \multirow{2}{*}{ Sexo } & Mujer & $5.5 \%$ & 8 & $71.7 \%$ & 104 & $22.7 \%$ & 33 & \\
\hline & Hombre & $0 \%$ & 0 & $73 \%$ & 44 & $27 \%$ & 16 & \\
\hline \multirow{3}{*}{ Edad } & 15 a 20 & $3 \%$ & 6 & $71 \%$ & 128 & $26 \%$ & 46 & \\
\hline & 21 a 25 & $8 \%$ & 2 & $76 \%$ & 19 & $16 \%$ & 4 & 0.446 \\
\hline & 26 a 36 & $0 \%$ & 0 & $100 \%$ & 3 & $0 \%$ & 0 & \\
\hline \multirow{2}{*}{$\begin{array}{l}\text { Años de } \\
\text { Estudio }\end{array}$} & 10 a 15 & $3 \%$ & 5 & $73 \%$ & 138 & $24 \%$ & 45 & \multirow{2}{*}{0.116} \\
\hline & 16 a 23 & $15.3 \%$ & 2 & $69.2 \%$ & 9 & $15.3 \%$ & 2 & \\
\hline \multirow{3}{*}{$\begin{array}{l}\text { Estado } \\
\text { Civil }\end{array}$} & Soltero (a) & $4 \%$ & 7 & $73 \%$ & 141 & $23 \%$ & 44 & \multirow{3}{*}{0.480} \\
\hline & Casado (a) & $0 \%$ & 0 & $0 \%$ & 0 & $0 \%$ & 0 & \\
\hline & Otro estado civil & $7 \%$ & 1 & $66 \%$ & 10 & $27 \%$ & 4 & \\
\hline \multirow{3}{*}{$\begin{array}{l}\text { Principal } \\
\text { Situación } \\
\text { Laboral }\end{array}$} & Estudiante & $4 \%$ & 7 & $72 \%$ & 137 & $24 \%$ & 45 & \multirow{3}{*}{0.571} \\
\hline & Trabajo remunerado & $0 \%$ & 0 & $70 \%$ & 7 & $30 \%$ & 3 & \\
\hline & Otra situación laboral & $12.5 \%$ & 1 & $63 \%$ & 5 & $25 \%$ & 2 & \\
\hline
\end{tabular}

Fuente: Elaboración propia. 


\section{DisCUSIÓN}

En los dominios relacionados con el Whodas 2.0, se logra identificar en el presente estudio; que la mayoría de los estudiantes muestran algún tipo de dificultad en los procesos cognitivos como: atención, concentración, memoria, evocación, aprendizaje, comprensión y resolución de problemas; lo cual, puede producir un efecto negativo en el desempeño y rendimiento académico de la población universitaria.

Lo anterior es confirmado por un estudio de una universidad de México, en el que se investigan los principios de neurociencia aplicados en la educación universitaria, lo cual indica que las principales funciones cognitivas que favorecen el aprendizaje son la atención y la motivación del estudiante. La primera a cargo del proceso de selección de la información dentro del sistema nervioso, siendo el elemento fundamental que articula todos los procesos cognoscitivos, dirigiendo y seleccionando la información que se va a procesar; y la segunda, permite obtener gratificación al ejecutar alguna actividad, incrementando la atención y el aprendizaje (Valerio, Jaramillo, Caraza y Rodríguez, 2016).

No obstante, menos de la mitad de la población en estudio revela limitación en el dominio de movilidad, esto puede estar relacionado a la etapa del ciclo evolutivo en el que se encuentran los estudiantes. En otro orden de ideas, gran parte de la población no presenta una discapacidad diagnosticada que limite e influya en su desplazamiento y movilidad en las actividades de la vida universitaria.

En ese mismo sentido, un estudio de estudiantes universitarios de La Araucanía (Chile), analiza los circuitos de movilidad en esta comunidad, argumenta que "la movilidad no sólo se encuentra en relación con el desplazamiento físico de las personas, sino que además hace referencia a las consecuencias y significados sociales, culturales y económicos, así como el conocimiento de las experiencias individuales" (Riquelme, 2017, p. 28).

Por ello, los estudiantes universitarios realizan diversos y heterogéneos desplazamientos; sin embargo:

Al residir en comunas periféricas planifican las movilidades cotidianas desde la oferta de transporte público disponible, lo cual reduce altamente la autonomía móvil. Se considera que al residir en lugares donde el acceso a espacios de interés es considerablemente delimitado, las posibilidades de movilidad se tornan determinantes para permitir el acceso a lugares de estudio, trabajo y recreación. Puesto que, mientras unos pocos poseen los medios para acceder a lugares de manera ágil, planificada y con un alto grado de autonomía, muchos otros dependen de actores intermediarios que les permita la conexión cotidiana a esos lugares (Riquelme, 2017, p. 46).

En otro orden de ideas, la mayoría de los estudiantes no manifiestan dificultades para la ejecución de actividades de cuidado personal, siendo este uno de los principales dominios que interviene de forma trasversal en la calidad de vida de los estudiantes; visto que, es una actividad innata de los seres humanos, que fortalece el autoconcepto y autoestima cuando se realiza de manera autónomo e independiente.

No obstante, es importante considerar los cambios en las prácticas cotidianas de los estudiantes de universidades colombianas a lo largo de su formación profesional. Dado que, contar con conocimientos acerca del autocuidado y la importancia de este en un estilo de vida saludable, no garantiza su ejecución diaria, pues existen diferentes factores que inciden en la apropiación de estos (Escobar, Mejía y Betancourt, 2017). 
En efecto, es un aspecto que puede influir en el ámbito de la educación superior, puesto que las prácticas relacionadas con el autocuidado pueden ser positivas o negativas, debido a las dinámicas que se generan en el proceso de formación profesional, "en tal sentido al iniciar la carrera, los estudiantes llegan con algunas prácticas de autocuidado, que pueden ser interrumpidas o abandonadas y se adquieren otras nuevas" (Escobar et al., 2017).

Respecto a las relaciones con otras personas, se evidencia que aproximadamente la mitad del alumnado presenta dificultad, en virtud que este dominio exige habilidades de comunicación efectiva, interacción y participación social; así como empatía, confianza en el otro e intercambio de información. Lo anterior, involucra un soporte emocional y social para la participación en las actividades inmersas en la vida universitaria.

Dadas las condiciones que anteceden, en las relaciones con otras personas es de gran importancia resaltar que la entrada a la educación superior, es un momento decisivo para la vida del estudiante, puesto que, implica un conjunto de estrategias para lograr la readaptación a una serie de alteraciones significativas, donde el universitario no solo debe generar nuevas maneras de pensar, sino también nuevas relaciones sociales; es decir, nuevas redes de soporte social, además son consideradas como herramientas para el comportamiento adaptativo, que permite a los estudiantes tratar con eficacia las demandas y afrontar diversas situaciones de la vida diaria (Ospina, Betancourt y Martínez, 2018).

Con relación a las actividades de la vida diaria, la mitad de la población estudiantil encuestada presenta dificultad leve para llevar a cabo dichas actividades; valorando que la transición de la educación básica media a la superior sugiere otras demandas y capacidades de los estudiantes, con el fin de emitir una respuesta efectiva a las situaciones que se presentan en este contexto.

Por consiguiente, se evidencia que la mayor parte de la población muestra dificultad leve, moderada y severa en el dominio participación en sociedad, consecuente a una condición de salud temporal o permanente; esto genera un desequilibrio en la capacidad y desempeño del estudiante para interactuar en su contexto universitario en igualdad de oportunidades.

Por lo que corresponde al nivel global de funcionalidad de los estudiantes de esta investigación, se logra identificar que la mayor parte de la población encuestada presenta dificultad leve en los dominios evaluados por el Whodas 2.0; los cuales están inmersos en la participación de las actividades de la vida universitaria, siendo esta una posible situación alarmante para las academias de educación superior relacionados con el ingreso, la continuidad y el egreso oportuno de los estudiantes.

Lo anterior, puede vincularse a dificultades en el proceso de asimilación al cambio, entre la educación básica a la educación superior y las exigencias o demandas de las instituciones; también a una pobre orientación vocacional, lo que puede limitar dicha permanencia.

En este sentido, una de las causas específicas que llevan al estudiante a abandonar sus estudios, es la ausencia de orientación vocacional; ya que, desarrolla un rol fundamental, pues al no contar con total claridad en este aspecto a la hora de iniciar sus estudios universitarios, las personas jóvenes desertan prematuramente. Por consiguiente, es 
importante señalar que la cifra de estudiantes que deserta de sus estudios superiores en Latinoamérica se acerca al 50\% (Torres, 2018). En concordancia con lo anterior, si los beneficios concedidos por la universidad se perciben como superiores a los costos personales en términos de esfuerzo y dedicación por parte del estudiantado, la decisión será continuar en la institución, en caso contrario desertará (Hernández-Jiménez, MoreiraMora, Solís-Salazar y Fernández-Martín, 2020). Por su parte, Aranzazu y Rojas (2018) afirman:

El mayor porcentaje de abandono se presenta en el primer año de estudio, las edades promedio de abandono oscilan entre 18 y 19 años, periodo en el cual la población estudiantil se encuentra en la llamada adolescencia tardía. Según los autores es en esta etapa de cambios donde se empieza a instaurar la autonomía tanto familiar como intelectual, lo cual conlleva en ocasiones a la toma errada de decisiones y por ende al descuido e indecisión académica ( $p$. 202).

Cabe resaltar que en la presente investigación no se encuentran factores sociodemográficos, relacionados con el nivel de funcionalidad de los estudiantes en un ambiente universitario; en vista de que en este estudio no se tienen en cuenta los factores que determinan el rendimiento académico de la población y otros factores personales como: grado de satisfacción con la profesión escogida, nivel socioeconómico, número de personas que conforman el núcleo familiar, lugar de residencia, nivel de estudio de los padres y programa académico en el que se halla inscrito.

Las variables anteriormente mencionadas, son relevantes para definir el nivel de funcionalidad de una población en estudio, puesto que estos aspectos según la literatura influyen e intervienen de manera divergente en la vida de los universitarios. Sin embargo, en esta investigación se considera al estudiante desde una perspectiva holística, en la cual se desempeña y participa en diferentes actividades en la vida universitaria y no solo su interacción en el área académica.

No obstante, otros estudios afirman que la problemática del bajo rendimiento académico está relacionada con aspectos como la edad de ingreso de los estudiantes a la universidad, porque usualmente los universitarios que forman parte de estos estudios se encuentran entre los 17 y 22 años, correspondiendo estas edades a la etapa de la adolescencia y adulto joven, estimándose que la mayoría son menores de 18 años (Contreras, Caballero, Palacio y Pérez, 2010).

\section{CONCLUSIONES}

En esta investigación se logró identificar los aspectos sociodemográficos como sexo, edad, número total de años estudiados, estado civil y principal situación laboral de los estudiantes de primer semestre 2018-1 de la facultad de salud y rehabilitación de una institución universitaria de la ciudad de Cali (Colombia); donde la mayor parte de los participantes fueron de género femenino.

De igual modo, se determinó que más de la mitad de los estudiantes están ingresando a la vida universitaria en edades entre 15 y 19 años. Esto debido a su egreso de la educación básica a edades correspondientes en etapas de adolescencia o adultez temprana. 
Así mismo, se evidenció que la mayoría de la población estudiada, asumen como principal situación laboral el rol de estudiantes universitarios, por la gran demanda de tiempo que requieren las carreras del área de la salud y rehabilitación como lo son fisioterapia, terapia ocupacional y nutrición y dietética, para la formación profesional.

En lo correspondiente a los dominios evaluados a través del Whodas 2.0, se evidenció, que gran parte de la población en estudio presentaron dificultad en los procesos cognitivos. Por lo que se deben considerar, dado que son esenciales para favorecer el desempeño y provecho académico del estudiante universitario.

Además, menos de la mitad de la muestra tuvieron problemas para permanecer de pie por largos períodos de tiempo, ponerse de pie, moverse dentro de su hogar, salir de casa y caminar largas distancias. Lo que quizá se asocie a la adultez joven, siendo el curso de vida en el que se ubican los estudiantes.

Es preciso resaltar que la mayoría de los participantes no expresaron tener dificultades para ejecutar actividades de cuidado personal; lo cual es un punto a favor; ya que la realización de estas, de manera autónoma e independiente, le otorga al ser humano identidad ocupacional y bienestar integral, conduciendo a la adquisición de una mejor calidad de vida. Dado que, la realización de esta actividad le otorga sentido, valor y un lugar a la persona para ser reconocido en la sociedad como ser ocupacional.

En cuanto a relacionarse con otras personas, se logró reconocer que 44\% del alumnado manifestó dificultad leve para lograr interactuar con compañeros o grupo poblacional y 19\% mostró una mayor problemática a nivel moderado y severo. Cabe destacar, habilidades que se encuentran inmersas en este dominio, tales como relacionarse con personas que no conocen, mantener y preservar una amistad, tener una sana convivencia, hacer nuevos amigos y realizar actividades sexuales; se pueden ver afectadas generando dificultad en la participación de la vida universitaria. Igualmente, la mitad de la población en estudio reveló dificultad para realizar AVD, que comprende ocuparse de las responsabilidades domésticas, realizar y terminar las tareas del hogar de manera eficiente, ejecutar eficazmente las actividades académicas o cotidianas.

De la misma forma, la modificación de los patrones de ejecución, el cumplimiento de roles, el manejo del ocio y tiempo libre, participación social, la actividad de descanso y sueño, se ven irrumpidas por las diversas responsabilidades en ambiente universitario, dejando atrás intereses, amigos o aficiones recreativas. En atención a lo cual, el estudiante debe generar nuevas habilidades y capacidades de aprendizaje, sin contar la capacidad de adaptación y afrontamiento a la que es expuesto y debe desarrollar o mejorar.

En consecuencia, se halló que más de la mitad del alumnado manifestó dificultad en el dominio participación en sociedad, por presentar un estado de salud transitorio o duradero; lo que suscita un desequilibrio en el desempeño ocupacional de la persona, afectando la participación efectiva en el ambiente universitario, en el rol como estudiante, amigo, miembro activo en la sociedad. Los cuales son significativos para el individuo, considerando que estos aumentan la interacción con el entorno, favorecen el bienestar emocional y disminuye la deserción de los estudiantes de la educación superior.

Esto implica el autoconocimiento volitivo, es decir, el conocimiento que permite a una persona anticipar, elegir, experimentar e interpretar su comportamiento ocupacional. 
También las disposiciones (disfrutar, valorar y sentirse capaz de realizar una ocupación), así como las costumbres y autoconciencia (aquello que experimentamos al desempeñar una ocupación). Ambas componen lo que nos inclina hacia ciertas ocupaciones.

Referente al nivel global de funcionalidad de la población en estudio, se evidenció que la mayoría muestran dificultades leves en el nivel de funcionalidad de los dominios evaluados por el Whodas 2.0; lo que puede incrementar los porcentajes de deserción de los estudiantes principalmente en los primeros semestres.

Cabe señalar que en este estudio no se hallaron factores sociodemográficos, relacionados con el nivel de funcionalidad de la población en un ambiente universitario; dado que, en esta investigación no se consideraron los factores que determinan el rendimiento académico y otros factores personales anteriormente mencionados. Sin embargo, se valoró al estudiante desde una perspectiva holística e integral y su participación en las diferentes áreas de desempeño.

Por último, se concluye que es de gran importancia para las instituciones de nivel superior, el trabajo interdisciplinar de los profesionales de la salud como Fonoaudiología, Psicología, Trabajo Social y Terapia Ocupacional; ya que pueden aportar al desarrollo integral y disminuir o eliminar las diferentes barreras que un estudiante con o sin discapacidad pueda presentar durante el desempeño ocupacional en la vida universitaria. Asimismo, los aportes y el acompañamiento de los profesionales de salud se pueden brindar tanto a estudiantes como a las instituciones, permitiendo así que el derecho a la educación de calidad sea verdaderamente incluyente para cada persona.

\section{REFERENCIAS}

Acedo, C. y Opertti, R. (2012). Educación Inclusiva: de focalizar grupos y escuelas a lograr una educación de calidad como el corazón de una Educación para Todos (EPT). En, X. Rambla (Ed.), La Educación para Todos en América Latina. Estudios sobre las desigualdades y la agenda política en educación (pp. 23-44). Buenos Aires: Miño y Dávila.

Aranzazu, D. y Rojas, M. (noviembre, 2018). Aproximación a la deserción femenina del programa ingeniería administrativa en la facultad de minas de la universidad nacional de colombia 2010-2017. Presentado al Congreso CLABES VIII, Universidad Nacional, Bogotá, D.C., Colombia. Disponible en https://revistas.utp.ac.pa/index.php/ clabes/article/view/1926

Alharthi, M. (2019). First-Year Students' Adjustment to University Life: A Case Study of Implementing College Buddy Program. International Journal of Higher Education, 9(1), 116-125. https://doi.org/10.5430/ijhe.v9n1p116

Borzone, V. (2017). Autoeficacia y vivencias académicas en estudiantes universitarios. Acta Colombiana de Psicología, 20(1), 266-274. https://doi.org/10.14718/ACP.2017.20.1.13

Cancio-Bello Ayes, C, Lorenzo, A. y Alarcó, G. (2020). Autocuidado: una aproximación teórica al concepto. Informes Psicológicos, 20(2), 119-138. https://doi.org/10.18566/infpsic.v20n2a9 
Campos-García, A., Oliver, A., Tomás, J., Galiana, L. y Gutiérrez, M. (2018). Autocuidado: Nueva evidencia sobre su medida en adultos mayores. Revista Española de Geriatría y Gerontología, 53(6), 326-331. https://doi.org/10.1016/j.regg.2018.01.010

Contreras, K., Caballero, C., Palacio, J. y Pérez, A. (2010). Factores asociados al fracaso académico en estudiantes universitarios de Barranquilla (Colombia). Psicología desde el Caribe, (22), 110-135. Disponible en https://rcientificas.uninorte.edu.co/index.php/ psicologia/article/view/883

Cuenot, M. (2018). Clasificación internacional del funcionamiento, de la discapacidad y de la salud. Kinesiterapia-Medicina Física, 39(1), 1-6. https://doi.org/10.1016/S12932965(18)88602-9

Escobar, M., Mejía, A. y Betancur, S. (2017). Cambios en el autocuidado de estudiantes colombianos de enfermería, durante la formación profesional. 2015. Revistas Hacía la promoción de la salud, 22(1), 27-42. https://doi.org/10.17151/hpsal.2017.22.1.3

END. (2018). Memorias encuentro semilleros de investigación. [Vol. I]. Santiago de Cali: END. Recuperado de https://investigacion.endeporte.edu.co/images/cientifica/Memorias3.pdf

Garza, O. (2015). Presentación de las tareas, satisfacción de necesidades psicológicas básicas y bienestar en atletas universitarios. [Tesis Doctoral]. Universidad Autónoma de Nuevo León, Nuevo León, México. Disponible en http://eprints.uanl.mx/9299/

González, M. A. y Giraldo, S. (2019). Movilidad, transporte público, comunicación y cultura ciudadana en el margen del SITP-MIO en Santiago de Cali. [Proyecto de gra$d o]$. Universidad Autónoma de Occidente, Santiago de Cali, Colombia. Recuperado de http://red.uao.edu.co//handle/10614/12256

Gutiérrez, J. y Guzmán, G. (2017). Definición y prevalencia del trastorno cognitivo leve. Revistas Española de Geriatría y Gerontología, 52(1), 3-6. https://doi.org/10.1016/ S0211-139X(18)30072-6

Hernández-Jiménez, M. T., Moreira-Mora, T. E., Solís-Salazar, M. y Fernández-Martín, T. (2020). Estudio descriptivo de variables sociodemográficas y motivacionales asociadas a la deserción: la perspectiva de personas universitarias de primer ingreso. Revista Educación, 44(1), 1-19. https://doi.org/10.15517/revedu.v44i1.37247

Jirón, P. (2015). La movilidad como oportunidad para el desarrollo urbano y territorial. En, A. Cornejo, La ciudad que queremos (pp. 47-62). Santiago: Cámara de Diputados. Disponible en https://repositorio.uchile.cl/handle/2250/133112

Menéndez, C., Pevida, M. y Dopico, E. (2020). Educación inclusiva: ¿Por dónde empezamos? En, J. Díez y J. Rodríguez (Dirs.), Educación para el Bien Común: hacia una práctica crítica, inclusiva y comprometida socialmente (pp. 326-334). Barcelona: Octaedro. Recuperado de https://octaedro.com/wp-content/uploads/2020/02/16215-Educacion-para-el-Bien-Comun.pdf

Moriña, A., Cortes, M. y Molina, V. (2015). Educación inclusiva en la enseñanza superior: soñando al profesorado ideal. Revista latinoamericana de Educación Inclusiva, 9(2), 161-175. Disponible en http://www.rinace.net/rlei/numeros/vol9-num2/art9.html 
Moreno, J. E., Chiecher, A. C. y Paoloni, P. V. (2020). Trayectorias de ingresantes universitarios y estrategias de aprendizaje: sus implicancias en el rendimiento académico. Revista Educación, 44(2), 1-19. https://doi.org/10.15517/revedu.v44i2.40055

Olivera, C., Salvador, R. y Elorduy T. (2015). Actividades de la vida diaria. Productos de apoyo. En, F. Montagut, G. Flotats y E. Lucas (Ed.), Rehabilitación domiciliaria: principios, indicaciones y programas terapéuticos (pp. 90-103). Barcelona: Elsevier Masson.

OMS. (2015). Medición de la salud y la discapacidad: manual para el cuestionario de evaluación de la discapacidad de la OMS: WHODAS 2.0. Ginebra: Servicio Nacional de Rehabilitación. Disponible en https://apps.who.int/iris/handle/10665/170500

Ospina, M., Betancour, S. y Martínez, A. (2018). Recursos personales y redes de apoyo en el tránsito del joven a la universidad. Revista hojas y hablas, (15), 100-115. https:// doi.org/10.29151/hojasyhablas.n15a6

Parra, C. (2011). Educación Inclusiva: Un modelo de diversidad humana. Revista Educación y desarrollo social, 5(1), 139-150. Disponible en https://revistas.unimilitar.edu. co/index.php/reds/article/view/897

República de Colombia. Congreso de la República. (28 de diciembre de 1992). Por la cual se organiza el servicio público de la Educación superior. [Ley 30]. Diario Oficial No. 40.700. Disponible en https:/www.mineducacion.gov.co/1621/article-86437.html

Riquelme, H. (2017). Cruzando la Arauca (Chile). Un análisis de las prácticas de movilidad cotidiana de universitarios adscritos a la gratuidad. Cultura-Hombre-Sociedad, 27(1), 23-50. http://dx.doi.org/10.7770/cuhso-v27n1-art1131

Rivera, M., Martínez, M., González F. y Salazar, A. (2016). Autoeficacia, participación social y percepción de los servicios universitarios según el sexo. Revista de psicología (Santiago), 25(2), 1-16. http://dx.doi.org/10.5354/0719-0581.2016.44842

StataCorp. (2011). Stata Statistical Software: Release 12. College Station: StataCorp LP. Disponible en www.stata.com/support/faqs/resources/citing-software-documentationfaqs/

Torres, I. (Noviembre, 2018). Estudio Longitudinal Permanencia y Abandono en Universitarios (2015- 2019). Presentado al VIII Congresos CLABES, Conferencia Latinoamericana sobre el Abandono en la Educación Superior, Ciudad de Panamá, Panamá. Recuperado de https://revistas.utp.ac.pa/index.php/clabes/article/view/2050

Üstün, T. B., Kostanjsek, N., Chatterji, S., Rehm, J. \& World Health Organization. (2010). Measuring health and disability: Manual for WHO disability assessment schedule WHODAS 2.0. Genebra: World Health Organization. Available: https://apps.who.int/ iris/handle/10665/43974

Valerio, G., Jaramillo, J., Caraza, R. y Rodríguez, R. (2016). Principios de Neurociencia aplicados en la Educación Universitaria. Formación Universitaria, 9(4), 75-82. http:// dx.doi.org/10.4067/S0718-50062016000400009 
Velázquez, D. E. (2019). Procesos cognitivos en estudiantes del primer año de la carrera ingeniería agropecuaria de la facultad de ciencias agropecuarias y desarrollo rural de la universidad nacional de Pilar. Brazilian Journal of Development, 5(1), 16541667. Disponible en https://www.brazilianjournals.com/index.php/BRJD/article/ view/1152/1014

Sandra Marcela Zúñiga Bolívar es Terapeuta Ocupacional. Docente investigadora y líder del grupo INVENDTO. Experta en tematicas relacionadas con la salud publica y la discapacidad. Docente de cursos teóricos y practicos. ORCID: https://orcid.org/0000-00034931-3369

Faisury Mejía Botina es profesional en Terapeuta Ocupacional. Egresada de la Institución Universitaria Escuela Nacional del Deporte. Realizó intervención en el sector penitenciario por 4 meses en calidad de pasantía profesional. Desde el 2020 a la actualidad se desempeña como contratista de apoyo en el servicio de Psicología de la Institución Universitaria Escuela Nacional del Deporte, en la cual brinda atención y orientación de Terapia Ocupacional de manera individual y grupal a la comunidad estudiantil. ORCID: https://orcid.org/PEP0002-8978-7457

Daniela Andrea Grijalba Pantoja es Terapeuta Ocupacional. Ex integrante del grupo de investigación INVENDTO. Actualmente partícipe del área de salud mental de Coserssa IPS SAS y apoyo en procesos de auditoría y seguimiento de casos en salud. Diplomada en neuropsicología de trastornos del aprendizaje y del lenguaje de la Universidad del Rosario (Colombia). Actualmente, estudiante de ultimo semestre de la especialización Gerencia en Servicios de Salud de la Universidad Libre (Seccional Cali, Colombia). ORCID: https:// orcid.org/0000-0001-9191-1544

María Camila Gamboa Gómez es Terapeuta Ocupacional con desempeño en el area de hospital en casa, con manejo de pacientes Neurológicos. ORCID: https://orcid.org/00000001-9917-7727 